EPV277/\#126 A CROSS-SECTIONAL, NON-INTERVENTIONAL, MULTICENTRIC STUDY TO DETERMINE THE PREVALENCE OF HOMOLOGOUS RECOMBINATION DEFICIENCY AMONG WOMEN WITH NEWLY DIAGNOSED, HIGH-GRADE, SEROUS OR ENDOMETRIOID OVARIAN CANCER

${ }^{1} \mathrm{C}$ Malaga*, ${ }^{2} \mathrm{M}$ Mokhtar, ${ }^{2} \mathrm{H}$ Azim, ${ }^{3} \mathrm{~A}$ Tyulyandina, ${ }^{4} \mathrm{C}-\mathrm{H}$ Wu, ${ }^{5} \mathrm{~L}-\mathrm{S}$ Yeh, ${ }^{6} \mathrm{~W}-\mathrm{S}$ Liou, ${ }^{7} \mathrm{~W}-$ F Cheng, ${ }^{8} \mathrm{~A}$ Chao. ${ }^{1}$ AstraZeneca, Medical, San Jose - Cost Rica, UK; ${ }^{2}$ Kasr El Ainy School of Medicine - Cairo University, Medical Oncology, Cairo, Egypt; ${ }^{3}$ N.N. Blokhin National Medical Research Center of Oncology, Medical Oncology, Moscow, Russian Federation; ${ }^{4}$ Kaohsiung Chang Gung Memorial Hospital, Gynecology, Kaohsiung City, Taiwan; ${ }^{5}$ China Medical University Hospital, Gynecology, Taichung, Taiwan; ${ }^{6}$ Kaohsiung Veterans General Hospital, Gynecology, Kaohsiung City, Taiwan; 'National Taiwan University Hospital, Gynecology, Taipei City, Taiwan; ${ }^{8}$ Chang Gung Memorial Hospital-Linkou, Gynecology, Taoyuan City, Taiwan

\subsection{6/ijgc-2021-IGCS.348}

Objectives Background Homologous recombination deficiency (HRD) is common in women with newly diagnosed high-grade serous ovarian and other morphologically related cancers. Exploiting the prevalence of HRD positive status can help optimize the use of targeted therapies in these patients and improve survival. Due to limited statistics, the study is aimed to determine global as well as country-specific (Egypt, Lebanon, Malaysia, Russia, Singapore, Taiwan, Saudi Arabia, Turkey, and United Arab Emirates) data on the prevalence of HRD positive patients using locally developed tests and commercial kits.

Methods Method Study design and population: This cross-sectional, non-interventional, multicentre observational study will enroll a minimum of 405 women ( $\geq 18$ years) with highgrade serous or endometrioid ovarian, primary peritoneal, and/or fallopian tube cancer having histopathology report and formalin-fixed paraffin-embedded (FFPE) tumour tissue block (s). FFPE tissue blocks will be used for HRD status and BRCA mutation testing. Objective: Primary endpoints include prevalence of patients with positive HRD status. Secondary endpoints include 1) Region- and country-specific prevalence of the patients with a) positive HRD status, b) positive tBRCA1m/tBRCA2m; 2) risk factors associated with these patients. Exploratory endpoints include clinical characteristics of overall patient population and by geographical regions. Statistical analysis: Analyses will be performed using full analysis set (FAS). Logistic regression analysis will be used to identify the potential risk factors.

Results Trail in progress.

Conclusions Importance The study will generate reliable evidence on prevalence of HRD positive patients and help health care professionals to understand the clinical and genetic characteristic of the disease in various countries guiding optimal treatment.

\section{EPV278/\#248 A RANDOMIZED CONTROLLED PHASE II CLINICAL TRIAL OF APATINIB PLUS CHEMOTHERAPY IN THE FIRST-LINE(1L) TREATMENT OF IVB STAGE, RECURRENT OR PERSISTENT CERVICAL CANCER}

Y Zhou, Y Jiang, M Zheng*. Sun Yat-sen University Cancer Center, Gynaecology Department, Guangzhou, China

10.1136/ijgc-2021-IGCS.349
Objectives This study evaluates the efficacy and safety of apatinib combined with chemotherapy in cervical cancer.

Methods In this open-label phase II study, eligible patients were randomized(1:1) to receive treatment: Arm A) oral apatinib $375 \mathrm{mg}$ once daily + intravenous paclitaxel (P) and cisplatin (DDP) or carboplatin (carb); Arm B) intravenous P and DDP or carb. Chemotherapy was administered for 4-6 cycles followed by apatinib. The primary endpoint was PFS assessed by RECIST1.1, and secondary endpoints included OS, ORR, DCR, DOR, and safety/tolerability.

Results 61 patients were randomized to Arm A $(\mathrm{n}=31)$ and Arm B (n = 30). Until March 30, 2021, the median followup was 10.7 months (range 1.73-32.23). Compared with patients in Arm B, patients in Arm A showed a higher PFS (13.6 vs. 5.2 months, HR, 0.455 ; 95\% CI 0.239-0.865; P = $0.014)$, ORR $(58.10 \%$ vs. $23.3 \%)$, and DCR $(80.60 \%$ vs. 53.3\%). Treatment-related AEs (TRAEs) occurred in $87 \%$ (A) and $40 \%$ (B); Grade $\geq 3$ TRAEs occurred in $77 \%$ (A) and $30 \%$ (B), respectively. The most commonly reported grade $\geq 3$ AEs were hematologic in nature (eg, neutropenia) and consistent with known chemotherapy AEs. Serious TRAEs were reported in 8 patients $(22.6 \%[\mathrm{~A}]$; $3.3 \%[\mathrm{~B}])$; TRAE leading to death was reported in 1 patient in Arm A.

Conclusions As 1L treatment of IVB stage, recurrent or persistent cervical cancer, the addition of apatinib to chemotherapy significantly improved PFS and showed a higher ORR and DCR than chemotherapy alone. No new safety issues were identified with the addition of apatinib to chemotherapy.

\section{EPV279/\#351 EPIK-0/ENGOT-OV61: A PHASE 3, RANDOMIZED STUDY OF ALPELISIB + OLAPARIB IN PATIENTS WITH NO GERMLINE BRCA MUTATION DETECTED, PLATINUM-RESISTANT OR -REFRACTORY, HIGH- GRADE SEROUS OVARIAN CANCER}

${ }^{1} \mathrm{P}$ Konstantinopoulos* ${ }^{2} \mathrm{~A}$ González-Martín, ${ }^{3} \mathrm{~F}$ Cruz, ${ }^{4} \mathrm{M}$ Friedlander, ${ }^{5} \mathrm{R}$ Glasspool, ${ }^{6} \mathrm{D}$ Lorusso, ${ }^{7} \mathrm{C}$ Marth, ${ }^{8} \mathrm{~B}$ Monk, ${ }^{9} \mathrm{~J}-\mathrm{W}$ Kim, ${ }^{10} \mathrm{D}$ Alsadius, ${ }^{10} \mathrm{~V}$ Pretre, ${ }^{10} \mathrm{Y}$ Han, ${ }^{1} \mathrm{U}$ Matulonis. ${ }^{1}$ Dana-Farber Cancer Institute, Medical Oncology, Boston, USA; ${ }^{2}$ Clínica Universidad de Navarra, Department of Medical Oncology, Madrid, Spain; ${ }^{3}$ Instituto Brasileiro de Controle do Câncer, Clinical Research Center, Sao Paolo, Brazil; ${ }^{4}$ University of New South Wales, Medical Oncology, Sydney, Australia; ${ }^{5}$ Beatson West of Scotland Cancer Centre and University of Glasgow, Clinical Trials Unit, Glasgow, UK; ${ }^{6}$ Fondazione Policlinico Universitario A Gemelli IRCCS, Gynaecology Oncology Unit, Milano, Italy; ${ }^{7}$ Medizinische Universität Innsbruck, Department of Obstetrics and Gynecology, Innsbruck, Austria: ${ }^{8}$ University of Arizona, College of Medicine, Phoenix, USA; ${ }^{9}$ Seoul National University, Obstetrics and Gynecology, Seoul, Korea, Republic of; ${ }^{10}$ Novartis Pharmaceuticals Corporation, Global Medical Affairs, Oncology, East Hanover, USA

\subsection{6/ijgc-2021-IGCS.350}

Objectives High-grade serous ovarian cancer (HGSOC) represents most epithelial ovarian cancers. Whilst initially responding to platinum-based therapy, $\sim 75 \%$ of patients develop resistance, conferring poor prognosis. Homologous recombination repair proficiency is associated with platinum resistance and limited response to PARP inhibitors. PI3K pathway inhibition downregulates BRCA expression, abrogating homologous recombination repair proficiency, and may lead to (re)sensitization to PARP inhibitors. As alpelisib (PI3K $\alpha$ inhibitor) + olaparib (PARP inhibitor) demonstrated preliminary synergism in platinum-resistant/refractory, BRCA-wild-type, recurrent HGSOC in a phase $1 \mathrm{~b}$ study, the EPIK-O study is further evaluating this combination. 